\title{
Le quartier épiscopal de Byllis (Albanie). Les campagnes de fouilles 2011-2012
}

Nicolas Beaudry, Pascale Chevalier, Tony Kozelj, Skënder Muçaj et Manuela Wurch-Kozelj

\section{(2) OpenEdition}

1 Journals

\section{Édition électronique}

URL : https://journals.openedition.org/cem/13268

DOI : $10.4000 /$ cem.13268

ISSN : 1954-3093

Éditeur

Centre d'études médiévales Saint-Germain d'Auxerre

\section{Référence électronique}

Nicolas Beaudry, Pascale Chevalier, Tony Kozelj, Skënder Muçaj et Manuela Wurch-Kozelj, « Le quartier épiscopal de Byllis (Albanie). Les campagnes de fouilles 2011-2012 », Bulletin du centre d'études médiévales d'Auxerre | BUCEMA [En ligne], 17.2 | 2013, mis en ligne le 13 décembre 2013, consulté le 22 septembre 2022. URL : http://journals.openedition.org/cem/13268 ; DOI : https:// doi.org/10.4000/cem. 13268

Ce document a été généré automatiquement le 22 septembre 2022.

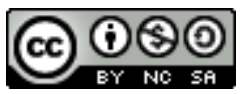

Creative Commons - Attribution - Pas d'Utilisation Commerciale - Partage dans les Mêmes Conditions 4.0 International - CC BY-NC-SA 4.0

https://creativecommons.org/licenses/by-nc-sa/4.0/ 


\title{
Le quartier épiscopal de Byllis (Albanie). Les campagnes de fouilles 2011-2012
}

\author{
Nicolas Beaudry, Pascale Chevalier, Tony Kozelj, Skënder Muçaj et \\ Manuela Wurch-Kozelj
}

1 Désertée à la fin $d u \mathrm{VI}^{\mathrm{e}}$-début du VII ${ }^{\mathrm{e}}$ siècle devant la pression continue des incursions avaro-slaves, la ville de Byllis, fondée à l'époque hellénistique au sommet d'une colline calcaire dominant la rive droite de la Vjosa (Aôos), constitue un observatoire privilégié des bouleversements que connaît le tissu urbain aux $\mathrm{V}^{\mathrm{e}}-\mathrm{VI}^{\mathrm{e}}$ siècles apr. J.-C. Siège épiscopal indépendant

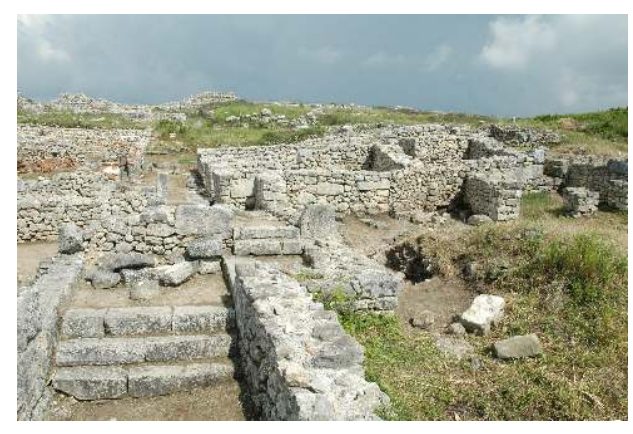
depuis le milieu $\mathrm{du} \mathrm{V}^{\mathrm{e}}$ siècle, c'était au $\mathrm{VI}^{\mathrm{e}}$ siècle l'une des villes les plus importantes de la province d'Epirus Nova. Ces deux siècles sont marqués par d'importants travaux de fortification au milieu du $\mathrm{VI}^{\mathrm{e}}$ siècle par l'architecte de Justinien Viktôrinos, réduisant de deux tiers l'espace intra-muros (11 ha) et provoquant une modification notable du réseau viaire, et la construction de thermes, d'ateliers et de maisons, ainsi que de cinq basiliques, dont une cathédrale sur le flanc de laquelle se concentrent les bâtiments nécessaires à la vie de l'évêché, qui finit par administrer cette petite cité montagnarde (fig. 1). 
Fig. 1 - Plan de la ville hellénistico-romaine avec la muraille, la voirie et les monuments tardoantiques et protobyzantins (๔ EfA, Manuela Wurch-Kozelj, 2012).

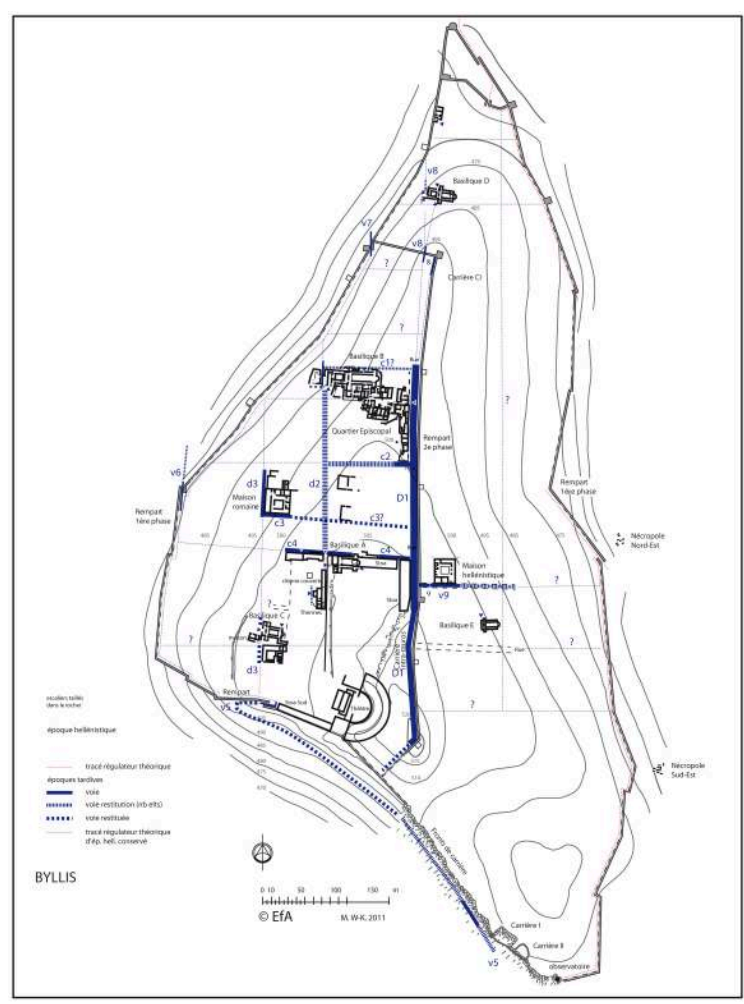

2 Les deux campagnes évoquées ici marquent la fin d'un projet bilatéral franco-albanais pluriannuel porté par l'École française d'Athènes et le ministère des Affaires étrangères et Européennes, bénéficiant d'un apport conséquent du Fonds québécois pour la recherche scientifique et culturelle et de l'université du Québec à Rimouski, en collaboration avec l'Institut archéologique du Centre des études albanologiques de la République d'Albanie et le Parc archéologique national de Byllis ${ }^{1}$. Les treizième et quatorzième campagnes sur le site de Byllis ont occupé chacune trois semaines, du 9 au 28 mai 2011 et du 11 au 28 mai $2012^{2}$. Elles visaient à terminer la fouille du quartier épiscopal de la cité bylliote, par quelques sondages et, en 2012, par une analyse architecturale de son extrémité sud-ouest (bâtiment D). Les travaux de 2011 ont compris le nettoyage de l'installation viticole $\mathrm{N}$, la poursuite d'opérations menées en 2009-2010 dans ce bâtiment et à la façade sud du bâtiment K-P, le dégagement du mur est de N5 ainsi que l'ouverture d'une tranchée transversale dans le passage N6, à des fins de consolidation des structures exposées. Une semaine a également été consacrée au nettoyage fin des maçonneries de la cathédrale et des annexes liturgiques pour en permettre le relevé photographique et en pierre-à-pierre et favoriser les discussions in situ de l'équipe. En 2012, l'examen s'est concentré enfin sur le bâtiment D et sur ses abords, mais aussi sur les nombreuses installations hydrauliques d'un secteur fouillé avant 1991 et dont l'étude systématique reste à parfaire. On a tant en 2011 qu'en 2012 poursuivi l'étude et la conservation des structures exposées dans ce secteur depuis 2000, et le traitement des échantillons archéo-environnementaux. Enfin, un scanner 3D portatif ViuScan prêté par le Laboratoire d'archéologie et de patrimoine de l'UQAR a été testé par N. Beaudry en 2011 sur des éléments de mobilier lithique conservés à Apollonia et sur le site. Le post-traitement, réalisé à Rimouski, permet d'apprécier les limites et le potentiel de cet outil sur un site comme Byllis. 


\section{Aperçu géomorphologique du site de Byllis}

Coraline Vinos-Poyo a conduit en 2011 une première approche géomorphologique du site de Byllis. L'environnement géographique montagneux du site de Byllis, qui prend position à environ $500 \mathrm{~m}$ d'altitude sur une terrasse calcaire de formation orogénique, est le paysage de la région de Mallakastër, traversé par la Vjosa, où l'on observe des formations collinaires dues au déchirement de la croûte terrestre par l'activité sismique et par la formation de cônes d'éboulis. Ces deux aspects (alluvionnement et colluvionnement) sont les points essentiels de l'évolution du paysage régional. Le plateau s'organise en plusieurs niveaux sur lesquels le site s'est implanté de façon hiérarchique, l'ensemble monumental (théâtre, stade, agora) occupant la terrasse méridionale surplombant la vallée de la Vjosa. L'accès au plateau se fait aujourd'hui par la face nord, mais des sentiers de pâturage existent sur le versant ouest, malgré sa pente raide, et continuent vers l'est par la route des crêtes qui permet de rejoindre à pied le site de Nikaia (Klos); seul le versant sud apparaît comme totalement inaccessible.

4 La formation montagneuse calcaire datant du Néogène a subi une activité sismique importante qui a fractionné le relief, entraînant la création de couloirs d'écoulement. La structure même de la roche constituant la région a favorisé ce phénomène et conditionne aujourd'hui encore le système érosif à la fois fluvial et de versant. L'importante activité permanente de l'érosion du plateau rend donc particulièrement difficile à retranscrire l'environnement précis, dans un paysage mouvant. Le deuxième facteur essentiel de l'évolution paysagère est la formation de la vallée alluviale de la Vjosa. Le fleuve se présente sous la forme d'un système en tresse à chenaux extrêmement mobiles, entraînant la modification du panorama d'une année sur l'autre. Cette mobilité au sein de la plaine alluviale induit des mouvements de sédiments différents en termes de déposition et d'érosion. Des vestiges d'anciennes terrasses alluviales sont observables en plusieurs points : sur le versant est, à environ $400 \mathrm{~m}$ d'altitude, on note une formation de sables et limons calcaires en litages alternant avec des passes à galets ; puis environ $300 \mathrm{~m}$ plus bas, sur le versant sud, un second niveau, au sein d'une carrière. Son intérêt particulier s'explique par sa diversité de niveaux alluviaux ainsi que par la présence au sein d'un faciès concrétionné de tessons de céramique d'un à deux centimètres. Ces deux témoignages de probables terrasses alluviales indiquent que la Vjosa a creusé son lit au sein des plateaux calcaires jusqu’à son cours actuel.

5 Le calcaire affleurant à Byllis est de type marneux, non karstique. Subissant les agents chimiques et climatiques, il se transforme en un sable limoneux brun qui recouvre l'intégralité du site. Au bas des versants apparait une autre forme de cette dissolution : une désagrégation rocheuse créant une calcrète. On est donc soit sur un paléosol aujourd'hui recouvert par les colluvions sablo-limoneuses du plateau, soit sur un niveau de ruissellement des eaux qui ont encroûté cette partie du versant. Plus bas encore, on retrouve la roche affleurante, sous la forme d'un escalier naturel. Les agents exogènes ont petit à petit effeuillé la roche. Ce secteur très abrupt, où pâturent moutons et chèvres, reçoit peu de sédimentation due aux colluvions. La roche à l'air libre se retrouve soumise à la pluie, au gel, au vent et aux agents chimiques. Sous cette 
couche de roche en décomposition, on retrouve un niveau de sables fins à moyens avec inclusions de cailloux calcaires hétérométriques.

6 La présence d'une route carrossable descendant depuis la porte sud (5) de l'enceinte hellénistique de Byllis vers Nikaia - une autre agglomération des Bylliones située au village actuel de Klos - est attestée par une inscription monumentale $(3,05 \times 2,03 \mathrm{~m})$ taillée dans le calcaire à flan de falaise ${ }^{3}$ et formant un repère choisi, bien visible à défaut d'être lisible depuis l'ouest et le sud. Cette route réalisée vers 150 apr. J.-C. sur un décret des décurions et sur les deniers personnels d'un évergète, nommé Marcus Valerius Lollianus ${ }^{4}$, commandant de plusieurs unités équestres, devait permettre la circulation des véhicules et des personnes. Son tracé au sortir de la Colonia [Iulia Augusta] Byllidensis, qualifié dans le texte même d'escarpé et de dangereux - [...] viam pub[licam] quae a col(onia) Byllid(ensium) per Astacias ducit an[g]ustam fragosam [per]iculosamq(ue) [...] -, n'est plus visible aujourd'hui que sur quelques dizaines de mètres le long de la falaise, sous des fronts de carrière dont l'exploitation avait peutêtre cessé au moment de la mise en service de la voie. Il n'est d'ailleurs aucunement assuré, car la route principale vers Nikaia semble plutôt partir de la porte 3 sur le flanc est. Quoi qu'il en soit, la pente méridionale si spectaculaire de la colline de Byllis a connu depuis l'occupation du site de forts remaniements dus à l'érosion des parois calcaires, mais aussi à la solifluxion extrêmement présente sur tout le pourtour de la pointe sud. L'accès principal, montant en lacets depuis la plaine quelque $500 \mathrm{~m}$ plus bas, se faisait par le versant ouest, moins abrupt, moins dangereux et plus facilement aménageable. La route Apollonia-Byllis via Gurëzeza aboutissait à la porte 6 et était encadrée par la nécropole romaine - on trouve des tombes hellénistiques près de la porte 5 au sud et 3 à l'est.

\section{Le quartier épiscopal}

7 Ce secteur urbain cohérent, dépendant de l'évêché, s'étend au sud-est de la cathédrale. Dans son dernier état bâti, du VI ${ }^{\mathrm{e}}$ siècle, sa cour centrale L, créée au croisement de deux axes perpendiculaires par l'arasement de structures antérieures, dessert des espaces à vocations économique et résidentielle, dont certains ont été examinés plus avant en 2011-2012 à la faveur de sondages et d'analyse des élévations.

8 Aux abords sud-est du bâtiment K-P, l'axe transversal nord-sud est coupé tardivement par un mur oblique, qui associe moellons et parpaings massifs. Ce mur, qui recouvre en partie des structures antérieures arasées avant la construction du bâtiment K-P, fait un coude vers l'ouest à la hauteur de la pièce d'angle P3, ménageant un espace de circulation devant la façade méridionale du bâtiment K-P, encore en fonction. Dans sa partie orientale, l'implantation diagonale du mur coudé semble n'être déterminée que par le besoin de relier deux points rapidement et au moindre coût: l'angle d'une grande citerne tardive taillée dans le rocher à l'est ${ }^{5}$; la rencontre de quatre murs arasés à l'ouest en face de la porte de P3. L'appareil hétéroclite et l'absence de fondations de ce tronçon trahissent la hâte et l'économie. Bien qu'il ne soit pas attesté sur toute la longueur de la façade de P1, ce mur - qui portait peut-être un aqueduc sortant de la citerne? - semble limiter de facto le domaine épiscopal au sud-est dans le contexte d'insécurité de la seconde moitié $\mathrm{du} \mathrm{VI}^{\mathrm{e}}$ et du début du VII siècle, s'il n'appartient à un état encore plus récent. 


\section{Un chai épiscopal de la seconde moitié du vie siècle}

Le bâtiment $\mathrm{N}$ prolonge vers l'est la façade du groupe cathédral sur la cour L, jusqu'au large passage nord-sud N6. La fouille de ce bâtiment a livré depuis 2007 l'ensemble des éléments constitutifs d'un chai de la seconde moitié du $\mathrm{vI}^{\mathrm{e}}$ siècle ${ }^{6}$, de la production vinicole au stockage : la pièce $\mathrm{N} 2$ mitoyenne à l'ouest de la pièce orientale $\mathrm{N} 5$, contient une banquette maçonnée et les fragments de sept ou huit pithoi bitumés; la pièce voisine N3 comptait au moins quatre autres pithoi, ainsi qu'un mortier taillé dans un bloc antique inscrit. Le premier fouloir, situé dans l'angle sud-est de N5, se déversait vers le nord dans une baignoire où le moût peut décanter avant d'être transvasé dans quatre pithoi étanchés au bitume, alignés à l'est. Dans l'angle nord-est de la pièce, le jus pressé dans un second fouloir, également rectangulaire et doté d'un escalier externe, se vidait vers l'ouest dans un bassin de recueil ; celui-ci se déversait à son tour, à travers un mur de refend, dans une cuve de vinification située dans la pièce N3A. La maçonnerie de pierre et de briques de cette cuve de vinification - 1,29 m EO x 1,86 m NS ; prof. max. 1,56 m - émergeait du sol pour former une margelle saillante, adossée à l'est au mur mitoyen avec $\mathrm{N} 5$ et au nord, au mur extérieur. Elle comportait un escalier de deux degrés adossé dans son angle sud-ouest ${ }^{7}$. Une cupule semi-ellipsoïdale $-44 \mathrm{~cm}$ EO x $55 \mathrm{~cm}$ NS ; prof. env. $19 \mathrm{~cm}$ - était ménagée au fond, dans l'axe du déversoir du bassin de recueil oriental. Enfin, au nord, à l'ouest et au sud, des briques liées au mortier formaient une feuillure contribuant à maintenir en place un couvercle de bois - 1,55 m EO x 1,90 m NS. Les parois intérieures et le rebord de la cuve enduits d'un mortier hydraulique ont été rechapés à au moins deux reprises.

La fouille de la cuve de vinification s'est conclue en 2011 (fig. 2).

Fig. 2 - Vue zénithale de la cuve de vinification du chai épiscopal (seconde moitié du vie siècle), cl. N. Beaudry.

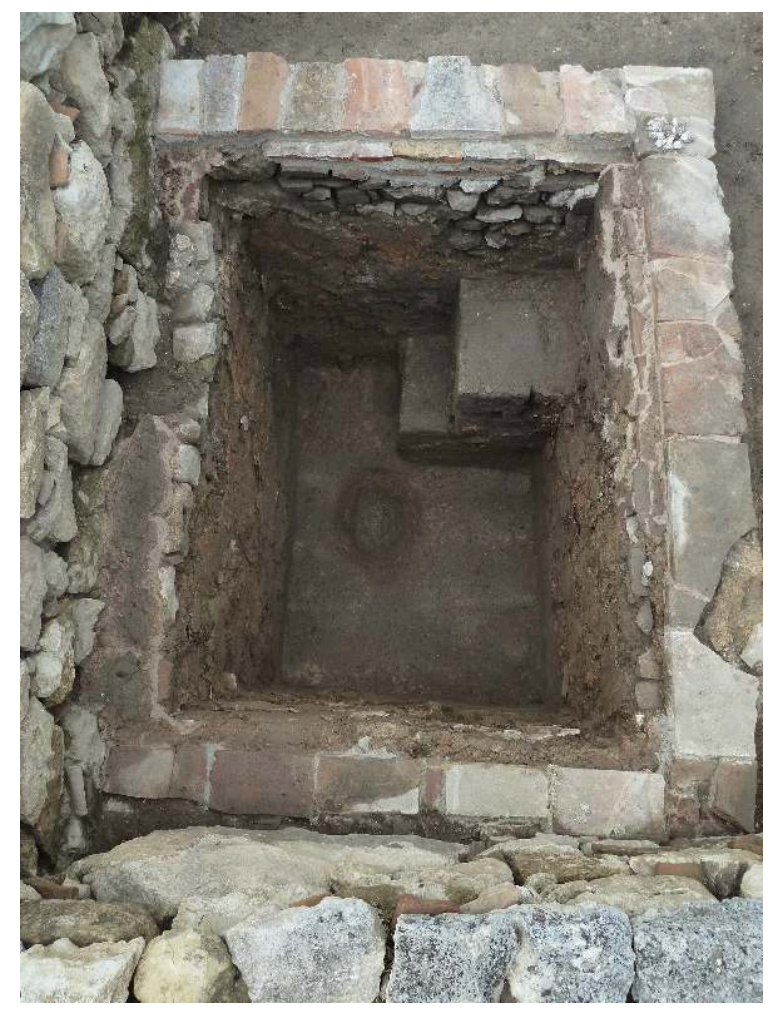


11 La priorité a ensuite été accordée par Agron Islami à la stabilisation préventive de ses vestiges en attendant une future présentation au public. Le bâtiment $\mathrm{N}$ est bordé à l'est par le passage N6, qui dessert la grande pièce N7 (à l'est) et forme la branche nord de l'axe de circulation transversal derrière la façade orientale du quartier et en contrebas de celle-ci. Comme N7, N6 a reçu des éboulis et des colluvions des structures en contrehaut; la pente vers l'ouest y est très forte. En prévision de la restauration du mur oriental de N5, très déformé par la pression des déblais, une tranchée large de 1,50 $\mathrm{m}$ a été ouverte sur toute la longueur du mur $(10,50 \mathrm{~m})$; une tranchée transversale (1 $\mathrm{m}$ x env. 3,50 m) a été implantée de façon à compléter une grande coupe est-ouest commencée en 2005. Elle n'a révélé qu'un sol argileux brun grisâtre et à l'ouest du sondage une courte portion d'égout associé, orienté NE-SO (fig. 3).

Fig. 3 - Coupe stratigraphique est/ouest à l'est du chai dans le passage N6 menant à la cour centrale du quartier épiscopal (P. Chevalier, DAO M. Chabrier).

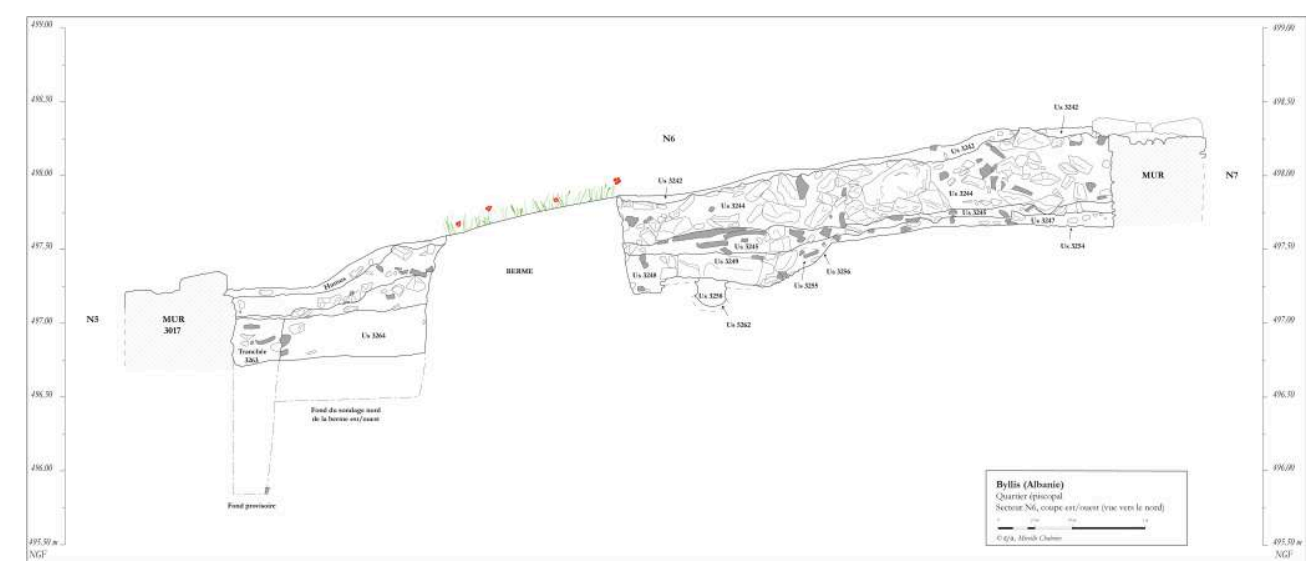

\section{Le bâtiment $D$ de la fin du $v^{\mathrm{e}}$ siècle}

Durant la campagne 2012, on a procédé à l'étude de bâti du bâtiment D au sud-ouest du quartier épiscopal (fig. 4). Comme celles du bâtiment K-P et des annexes les plus tardives de la cathédrale, les maçonneries mêlant pierre calcaire, blocs, morceaux de tuiles et de briques remployés, sont ici liées à la terre. Elles ne permettent pas d'effectuer d'analyses comparatives des mortiers pour préciser leur phasage relatif. L'examen minutieux in situ est par conséquent crucial. 
Fig. 4 - Le bâtiment tardif D vu depuis l'ouest (cl. N. Beaudry).

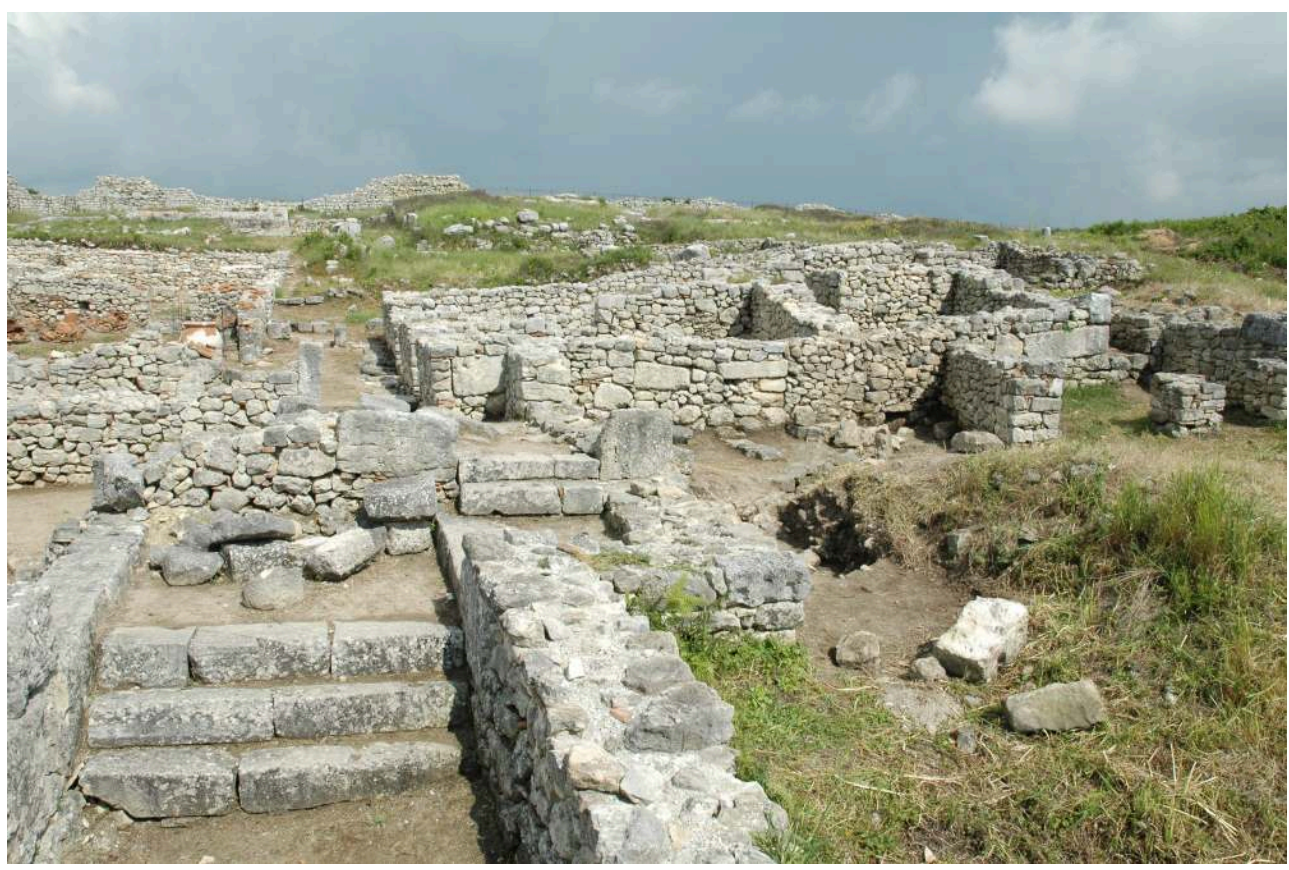

Sept pièces du bâtiment D ont été dégagées dans les années 1987-1991 par S. Muçaj ${ }^{8}$. L'ensemble se prolonge probablement vers le sud. Trois de ces pièces $(34,29$ et 27$)$ sont alignées vers le nord et empiètent largement sur l'ancienne voie décumane secondaire qui borde les annexes méridionales de la cathédrale. Face aux annexes 25, 21 et 33, cette façade « sur rue " méridionale du bâtiment $\mathrm{D}$ suit grossièrement l'axe est-ouest de la grille urbaine hellénistique et longe la paroi sud d'un cloaque tardif, en bordure de la rue réduite alors à un passage large de 2,20 à $3 \mathrm{~m}$. Un sondage transversal ouvert dans ce passage 26 a permis d'établir la stratigraphie et les liens avec l'égout, livrant plusieurs niveaux de circulation. L'interface supérieure du niveau qui scelle la tranchée de fondation de l'égout, a livré une lame de faux en fer. Sous des recharges de remblai argileux, un niveau de sol antérieur à l'égout est constitué d'un empierrement compact de calcaire jaune dans une matrice d'argile.

14 Les autres murs du bâtiment D dévient largement de l'ancienne trame urbaine, probablement afin de suivre le mur ouest du grand bâtiment K-P. Le bâtiment D s'implante en effet en équerre autour de l'angle sud-ouest de l'ensemble K-P, postérieurement à l'obturation des portes nord des pièces K4 et K5 et à la surélévation de leurs sols internes. Ainsi, le bâtiment D vient fermer à l'ouest la cour L par un mur orienté nord-sud, situé dans l'alignement presque exact du mur du chevet et du transept de la cathédrale. Ce mur s'appuie contre la façade nord du bâtiment K-P, au niveau du mur mitoyen des pièces $\mathrm{K} 4$ et $\mathrm{K} 5$. Dans son dernier état, l'accès à la cour L par le passage 26 est contrôlé par une porte, dont subsiste un des montants rapportés.

Contrairement à leurs voisines du bâtiment K-P, dont le plan régulier dénote une construction unitaire et une conception d'ensemble réfléchie, les pièces D34, 29, 27, 28 et le passage à escalier 30 sont de plans variés et présentent des irrégularités notables. Les espaces arrière D43 et 24 (à un niveau encore supérieur) et le portique ouest 41 constituent des adjonctions progressives. D34 consiste en un rectangle allongé dans le sens nord-sud de 2,80 x 6,50 m dans l'œuvre, fermé à l'ouest par un mur qui s'appuie sur le bouchage de la porte nord de K5. Lors de son édification, une très large ouverture 
$(2,50 \mathrm{~m})$ donne au nord sur le passage 26 ; elle est obturée dans second temps. Immédiatement à l'ouest, la pièce D29 arbore la forme d'un trapèze évasé vers le sud $(2,50 / 3,75$ x 4,20/5,30 m). À son angle sud-est, un court mur est construit sur $1,55 \mathrm{~m}$ de longueur nord-sud dans le prolongement du mur qui ferme la pièce K5 et le bâtiment K$\mathrm{P}$ à l'ouest ; le mur sud de la pièce D29 fait ensuite retour à angle droit, vers l'ouest et la pièce est close par un mur parallèle aux murs transversaux du bâtiment K-P - fermant le trapèze mentionné plus haut. Outre le recoin sud-est $(0,65 / 0,75 \times 1,55 \mathrm{~m})$, la pièce D29 comporte 4 portes, une d'accès au passage 26 au nord en face d'une porte menant à l'annexe 30, deux percées pratiquement en vis-à-vis dans les murs est et ouest et desservant les pièces 34 et 27 ; l'obturation de la porte ouest isolera la pièce D27. Cette dernière - un trapèze assez inégal de 3,50/4 x 3,70/4,10 $\mathrm{m}$ dispose à l'origine de deux autres portes : une à l'ouest vers la courette D42, également murée tardivement, et une au nord vers le passage 26, qui restera seule en fonction dans le dernier état. La pièce D27 ${ }^{9}$ est fermée au sud par un mur poursuivant de façon irrégulière le mur méridional de la pièce D29; une large banquette (haut. $46 \mathrm{~cm}$, prof. $62 \mathrm{~cm}$ ), composée d'un parement maçonné comprenant deux blocs équarris de bonne dimension, d'un blocage sec et d'une couverture de tuiles plates de toiture, court à ses pieds.

16 À l'arrière, au sud du bâtiment $\mathrm{D}$, on trouve à un niveau supérieur rattrapé par quelques marches une pièce grossièrement carrée D28 $(2 / 2,40 \times 2,50 / 2,70 \mathrm{~m})$ dont le volume prolonge celui de D27. Son mur oriental la sépare de l'espace de circulation et de passage mitoyen D30, qui contient un escalier assurant l'existence d'un étage.

17 Esquissons à présent une chronologie relative, débutant par les éléments qui ont précédé l'ensemble D (état A). Les constructions d'époque hellénistique, situées au sud de l'ancien decumanus, sont arasées : ne subsistent que quelques vestiges de murs et le tracé réorienté de l'égout central ${ }^{10}$. Deux "piliers » allongés, antérieurs au premier état du bâtiment $\mathrm{D}$, sont édifiés au-dessus du mur hellénistique arasé; avec à leurs extrémités de gros blocs forment chaînage. Ils servent ensuite dans les murs latéraux est et ouest de la pièce D27, expliquant son plan trapézoïdal évasé. L'espace s'étendant entre l'égout, dont le tracé suit grosso modo celui du cloaque médian de l'ancien decumanus et l'alignement de la façade nord du bâtiment voisin K-P, n'était donc pas libre avant qu'on y édifie le bâtiment $\mathrm{D}$. Dans sa phase finale, après la condamnation des portes de sa façade sur cour, le bâtiment K-P est remanié. L'écoulement du canal préexistant, qui longeait la façade nord des pièces $\mathrm{K} 4-\mathrm{K} 3$, est conservé un temps au sein du bâtiment $\mathrm{D}$, puisqu'on aménage un débouché dans le mur est de la pièce D34. Ce canal se poursuivait peut-être jusqu'au débouché du mur ouest de la pièce D28.

18 Les pièces D27, 20, 34, 28 et le passage 30, avec son massif d'escalier adossé, appartiennent bien à une campagne unitaire - état $B$-seconde moitié du $\mathrm{vl}^{\mathrm{e}}$ siècle. Les irrégularités du plan sont imputables à l'adaptation à l'espace disponible, à la déclivité nord-sud et aux éléments préexistants, notamment les piliers allongés intégrés dans la pièce D27 et l'angle du bâtiment K-P (déjà fortement réaménagé). Il est impossible de situer la construction de la banquette en D27, le bouchage du passage entre D26 et D34, l'obturation partielle du passage de D29 à D34, le bouchage de la porte menant de la courette 42 à la pièce D27, celui de la porte reliant D27 à D29 - tous ces travaux d'aménagement (état $C$ ) sont antérieurs aux états $\mathrm{D}$ à $\mathrm{F}$. Une extension s'accole au sud de l'ensemble, dans l'alignement de sa façade ouest à l'arrière de D28: la petite pièce D24 (état D), qui intègre un canal d'évacuation des eaux. Une extension en forme de portique sur piliers maçonnés s'appuie ensuite sur toute la façade ouest du bâtiment $D$ 
(état $E$ ) : son mur sud fait retour vers le nord et deux piliers sont alignés in situ; les terrassiers se rappellent avoir vu un troisième pilier au nord; ce portique menait probablement au passage 26. Un mur est-ouest cloisonne enfin le portique 41 en prolongeant le deuxième pilier - état $F$-début du $\mathrm{VII}^{\mathrm{e}}$ siècle ? -, il est possible qu'une autre cloison ferme l'accès nord au passage 26 .

On a donc là un bâtiment complexe, qui appartient à l'ultime phase du quartier et qui a lui-même été remanié à plusieurs reprises. Situé le long de l'axe de circulation principal du complexe et face à des annexes de la cathédrale ouvertes sur le passage hérité de la rue, séparé par une porte des annexes économiques et de l'habitat qui entourent la cour $\mathrm{L}$, il a des fonctions utilitaires et domestiques. S. Muçaj rapporte que les pièces en façade 27,28 et 34 ont livré des fragments de mosaïque pavimentale polychrome provenant de l'étage, plus grossière que celle des pavements de la cathédrale voisine (fig. 5).

Fig. 5 - Fragment de pavement de mosaïque de l'étage du bâtiment D (cl. N. Beaudry).

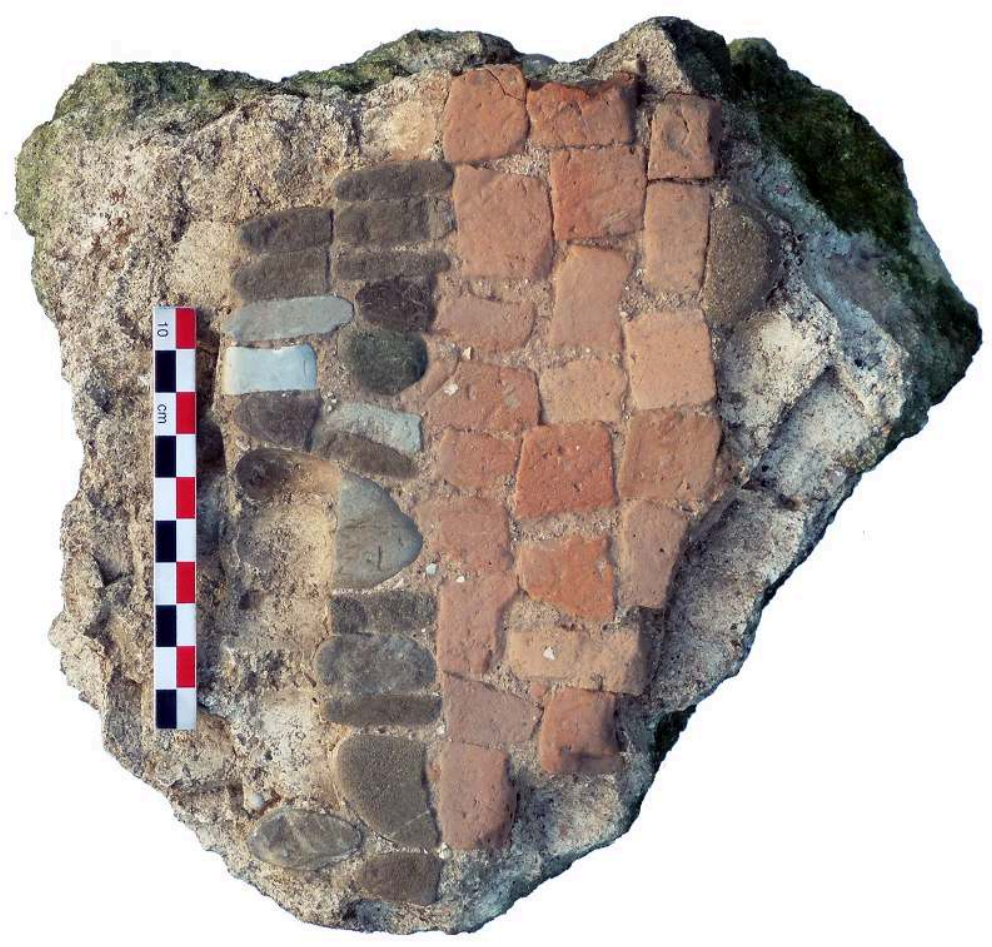

Les fenêtres géminées de l'étage étaient soutenues par des meneaux à impostes et bases, proches du point de vue de leur morphologie angulaire et du décor des impostes (croix et végétaux), des exemplaires de l'étage du bâtiment K-P. Ceci permet de croire que l'ensemble $\mathrm{D}$ avait une certaine dignité malgré la maladresse de son plan et ses maçonneries mixtes montées assez rapidement à la terre.

\section{La cathédrale}

Durant la campagne 2011, priorité a aussi été donnée à l'étude des phases de constructions de la cathédrale (basilique B) en vue de la publication Byllis 2. Une semaine a réuni Jean-Pierre Sodini, Pascale Chevalier, Marie-Patricia Raynaud, Tony et 
Manuela Kozelj, Elio Hobdari et Skënder Muçaj pour discuter in situ de problèmes variés : architecture des différents états de construction du début du $v^{\mathrm{e}}$ à la fin $d u \mathrm{VI}^{\mathrm{e}}$ siècle ; développements en plan, notamment des annexes ajoutées, de la tribune et des pièces à l'étage ; élévations restituables ; techniques de construction et de décor mural ; aménagements liturgiques successifs; pavements de mosaïque; sculpture architecturale et liturgique; liens avec le quartier épiscopal et le reste de la cité de Byllis...

La cathédrale s'est implantée légèrement en marge de l'agora antique sur des îlots d'habitation anciens, dont les structures rasées pour l'occasion se devinent sous les sols de mosaïque et dallages en place ou se lisent dans des portions d'élévation remaniées. Cette contextualisation des vestiges sur le temps long et des prémices de l'installation du groupe épiscopal ont constitué une première étape de travail. En fonction des vestiges antérieurs à l'époque paléochrétienne repérés, $\mathrm{M}$. Wurch-Kozelj a représenté les axes de circulation sur des plans, pour l'époque hellénistique puis pour la période qui suit la construction du rempart de Viktôrinos (fig. 1). L'identification de vestiges antérieurs à la construction de la cathédrale permet de préciser et de présenter les premiers éléments de répartition et d'aménagements des bâtiments antérieurs, qui mettent progressivement en évidence les données urbanistiques antiques (îlots, répartition dans l'îlot).

\section{L'annexe sud-est 38}

23 Un examen de la pièce 38 , dotée d'un beau pavement de mosaïque - la seule dans ce secteur - ainsi que d'un accès monumentalisé dans un second temps par un arc retombant sur des supports sculptés ressemblant à de grands meneaux de fenêtres, a été couplé avec des prélèvements de mortier - liants muraux, enduits de revêtement, fixation des bases, support de la mosaïque. Cet espace crucial dans l'organisation du complexe assure la liaison entre l'angle sud-est de la cathédrale et les zones à caractère économico-résidentiel du quartier épiscopal. S. Muçaj, qui l'a fouillée dans les années 1987-1991, y voit l'espace où l'on apporte et stocke certaines offrandes, l'huile pour le luminaire ou le vin de messe tiré des réserves épiscopales - ce qui justifie pleinement un apparat décoratif important.

24 On y a dégagé le pavement de mosaïque, libérant les bases des supports de l'arc nord qui reposent en partie dessus. Le nettoyage a permis un diagnostic sanitaire du pavement très affaissé et l'observation de deux couches d'enduits muraux crème chargé en paille dans les angles de la pièce et, de manière plus lacunaire, au pied des murs est et ouest. Les bases de l'arc sont contemporaines ou postérieures à la seconde couche d'enduit à la chaux. Une porte centrée à l'ouest est alors bouchée tandis qu'on en perce une à l'est, dans une dernière modification des circulations.

Les analyses de mortier effectuées par Stéphane Büttner en 2012 contribuent à la détermination fine du phasage de ces étapes de construction puis de modifications progressives, à ce point nodal de distribution des espaces sud-est du groupe cathédral, qui dessert également la cour ouverte au chevet derrière l'abside. Trois types de mortiers, tous tardifs dans le phasage général de la cathédrale, ont été reconnus et rapprochés d'échantillons déjà étudiés : l'un - issu du mur sud de l'annexe 37 (au nordest de 38), de l'enduit postérieur à la mosaïque dans l'angle sud-est de la pièce 38 et du dernier enduit de son mur ouest - correspond à un des deux types utilisés à la basilique 
E. Le deuxième type de mortier - bouchage de la porte nord de l'annexe 39, enduit postérieur à ce bouchage en 39 et dans le couloir 29 , joint de fixation sur la mosaïque d'un support de l'accès monumentalisé de la pièce 38 - se retrouve dans le dallage du transept - c'est-à-dire le dernier état du sanctuaire et du transept de la cathédrale -, dans le rempart protobyzantin du milieu du $\mathrm{VI}^{\mathrm{e}}$ siècle, ainsi que dans le mur gouttereau et la colonnade sud de la basilique E. Le troisième mortier - prélevé dans le mur sud du couloir 29 - est le même que celui des derniers enduits appliqués dans les zones septentrionales de la cathédrale et dans les deux annexes sud-ouest.

\section{Mosaïque et sculpture}

La présence sur le site de Marie-Patricia Raynaud et d'Agron Islami, venus retravailler sur certains détails de l'évolution complexe et de la mise en place des pavements de mosaïque, a donné lieu a plusieurs résultats pour le moins inattendus. En effet, le dégagement du pavement du tiers est du vaisseau central a laissé voir, dans une lacune du pavement le long du stylobate nord de la nef, une petite surface de tapis de mosaïque plus ancienne - dont se souvenait A. Islami. Son motif même, observé de manière fragmentaire, a permis à M.-P. Raynaud de restituer les dimensions du tapis d'époque romaine (III siècle ?) et pour T. et M. Kozelj de confirmer la répartition des pièces des domus antiques.

Outre l'amorce d'une étude synthétique de la sculpture, déjà cataloguée et dessinée par Elio Hobdari avec J.-P. Sodini et P. Chevalier suivant les diverses catégories de matériel - décor architectural, mobilier liturgique -, les dégagements des secteurs de mosaïques de la nef ont été l'opportunité de revoir précisément, avec J.-P. Sodini, P. Chevalier et T. Kozelj, la base de l'ambon. Un complément de relevé par T. Kozelj et une nouvelle description amendant et complétant un état de réfection tardive la restitution qui avait été proposée dans le Bulletin de correspondance hellénique $(B C H)$ de 2003, offrent quelques éléments nouveaux, notamment un modèle en 3D des deux états de l'aménagement liturgique $\mathrm{du} \mathrm{VI}^{\mathrm{e}}$ siècle. L'étude des phases de constructions ou plutôt d'extensions et/ ou de modifications ayant abouti à l'état construit final, que nous observons de la manière la plus évidente, est en cours, tant par écrit que de manière graphique. Des restitutions sont élaborées à partir des relevés et observations sur le terrain effectués ces dernières années et en 2011. Au terme de la campagne, quelques points ont encore été discutés par P. Chevalier, M.-P. Raynaud et J.-P. Sodini à Paris (mosaïque), puis avec Catherine Vanderheyde à Strasbourg (sculpture) en juin et septembre 2011. L'ensemble de ces éléments sera publié dans le volume Byllis 2, dont la campagne 2011 garantit l'avancement rapide. Les travaux récents ont été présentés au $22^{\text {nd }}$ International Congress of Byzantine Studies à Sofia fin août 2011 (P. Chevalier), puis en janvier 2012 à Philadelphie à l'Archaeological Institute of America $113^{\text {th }}$ Annual Meeting (N. Beaudry).

\section{Bâtiment antique et tardo-antique dit «Terentianum »}

Skënder Muçaj a mené en 2008-2012 la fouille d'un petit bâtiment de l'Antiquité tardive, situé au sud du quartier épiscopal, le long du flanc sud du cardo du rempart, entre la cathédrale et l'ancienne agora. La maison tardo-antique s'implante sur une domus romaine, elle-même surimposée à une maison hellénistique; la domus comporte une pièce contenant deux autels dédiés à deux de ses fils par leur père Terentius. Cette 
fouille complémentaire du programme franco-albanais permet de documenter dans l'architecture civile les grandes séquences chronologiques urbaines mieux connues pour les églises et le quartier épiscopal.

\section{Étude archéozoologique}

29 Au cours de la mission 2011, Jean Cantuel a analysé la faune provenant de sondages dans la cathédrale (2000-2001) ainsi qu'une partie des ossements recueillis dans le bâtiment K-P et le chai N - 3590 os déterminés sur un total de 6356 .

L'assemblage osseux issu de la cathédrale, où le taux de détermination est le plus important - 64,7\% contre env. $50 \%$ dans les autres corpus -, montre une composition où dominent (comme ailleurs) les caprinés $(47,5 \%)$, mais où les bovidés semblent mieux représentés $(18,4 \%)$ que d'habitude; les suidés représentant $27,8 \%$ et la faune aviaire $6,3 \%$. Outre quatre restes d'équidés, on observe aussi faiblement du chien, du lièvre, du chevreuil, du sanglier, de la tortue, de l'ichtyofaune, ainsi qu'une huitre (Ostrea edulis) et un murex (Hexaplex trunculus). Dans l'espace culinaire P1A-P1C, la faune découverte est similaire en termes d'espèces représentées, avec une fragmentation d'origine anthropique importante en $\mathrm{P} 1 \mathrm{C}$, où la faune aviaire est plus forte qu'en P1A. Les animaux de petite taille (chèvres, moutons et porcs) l'emportent nettement sur les bovins - seulement $2,2 \%$ des restes en P1C, 9,9\% en P1A et 4,9\% en P4. L'espace P1 permet de mieux appréhender les modes de préparation et de consommation de la viande. La quasi-absence de brûlures sur les ossements implique une cuisson indirecte de la chair animale, bouillie ou mitonnée dans un récipient. On note sur ces os des stries, soit courtes et fines de décarnisation au couteau sur les diaphyses d'os longs, soit très profondes sur les épiphyses des os longs ou sur l'acetabulum, résultant de la mise en quartiers de l'animal. Se confirme par là une double fonction de l'ensemble $\mathrm{P}$ : mise en pièces des carcasses et préparation/cuisson de quartiers de viande. On y a aussi recueilli un peu d'ichtyofaune et des coquillages - Patella sp., Spondylus gaederopus, Hexaplex trunculus.

31 Les restes fauniques sont abondants mais plus altérés dans le chai N : 1342 restes identifiés (taux de détermination $53 \%$ ). Les bovins y représentent 13-19\% de la faune domestique, les caprinés apparaissent moins nombreux que dans la cuisine $\mathrm{P}$, avec peu de faune aviaire. On remarque enfin en N3A une cinquantaine de restes issus de fotus de caprinés, suidés et canidés, ainsi qu'un os d'équidé et de chat.

32 Associée aux autres données archéologiques, l'étude en cours permettra à terme de mieux caractériser la fonction des espaces, les pratiques alimentaires, les modes d'élevage et leur but, et, au-delà, le mode de vie à Byllis aux époques paléochrétienne et protobyzantine.

\section{Étude du verre}

Le travail d'inventaire et de catalogue du mobilier en verre a été poursuivi en 2011 par Julie Viriot - 172 fragments soit plus d'une centaine d'objets. L'inventaire complet dénombre actuellement près de 400 fragments de verre issus de la basilique $B$ et du quartier épiscopal, ainsi que de la basilique $\mathrm{E}$, avec un beau fonds de verre de vitres. 

quartier épiscopal (Byllis $4^{11}$ ) a été planifiée pour les années prochaines. Elle sera dirigée par $\mathrm{N}$. Beaudry et mettra l'accent sur l'évolution tardive du quartier au vi siècle en tenant compte des fonctions économiques bien attestées sur les lieux et de l'apport des études paléoenvironnementales. Il revient à S. Muçaj la tâche de présenter les opérations archéologiques menées en 1987-1991 avant le début de la mission francoalbanaise. Le petit secteur concerné au sud-ouest, qui comprend le bâtiment d'habitation D à étage - le plus récent de l'ensemble -, est jointif avec les annexes liturgiques de la cathédrale; cette relation sera traitée par P. Chevalier au terme d'une présentation rapide de l'ecclesia faisant l'objet du volume Byllis 2.

le terme de l'ANR AdriAtlas, à Rome, en novembre 2013, une exposition plus complète de nos résultats se tiendra à la Galerie nationale de Tirana au printemps 2014, sous l'égide de l'ambassade de France. Nous avons aussi convenu de préparer pour la fin 2013 les nouveaux panneaux signalétiques en trois langues du Parc archéologique national de Byllis. Financés par l'ambassade de France à Tirana, ils intégreront notre documentation graphique et photographique ainsi que des restitutions 3D des basiliques et des stoai de l'agora. Le dernier guide touristique du site, paru en albanais et en anglais en 2009, puis en français en $2011^{12}$, sous la plume de Neritan Ceka et S. Mucaj, reprend déjà maints documents graphiques et photographiques issus de nos travaux, inédits par ailleurs pour certains.

Enfin, parallèlement aux articles de diffusion scientifique habituels, notre équipe a été associée au projet d'atlas informatique de l'Adriatique antique, AdriAtlas, soutenu par l'ANR et l'EFR et coordonné par Francis Tassaux (Bordeaux 3-Ausonius); P. Chevalier ayant en charge la coordination de l'ensemble des sites tardoantiques/haut-médiévaux de l'Atlas qui les prend en compte jusqu'à l'an 751. E. Hobdari se charge du recollement et du choix des sites tardoantiques/haut-médiévaux pertinents sur tout le territoire albanais ${ }^{13}$. La notice sur Byllis, rédigée par P. Chevalier, S. Muçaj et E. Hobdari, est en ligne sur le site depuis mai $2013^{14}$. Elle valorise de manière intéressante et 
partiellement inédite les acquis de la mission. Cet Atlas web insistant également sur la dimension de protection et de mise en valeur patrimoniale et touristique des sites recensés, la présence de Byllis en son sein favorisera les politiques de développement durable sur le site.

Reçu : 20 août 2013 - Accepté : 20 novembre 2013

\section{NOTES}

1. Cf. Bucema 12 (2008), p. 63-68 [cem.revues.org/index6512.html]; 13 (2009), p. 73-76 [cem.revues.org/index11233.html] ; 14 (2010), p. 57-60 [cem.revues.org/index11539.html] ; et 15 (2011), p. 91-95 [cem.revues.org/index11925.html].

2. L'équipe se composait ainsi :

- du côté albanais: Skënder Muçaj, Institut archéologique, QSA-Qendra e Studimeve Albanologjike, Tirana, (chef de mission) ; Elio Hobdari, architecte, docteur en archéologie (IAQSA) ; Skënder Bushi, dessinateur (Agjensia e Shërbimit Arkeologjik, Tirana) ; Olgita Ceka, doctorante (université de Stuttgart, Allemagne); Suela Xhyheri, doctorante (QSA); Geralda Resulaj, étudiante (université de Tirana); Irkild Ristani, architecte (Tirana); Agron Islami, restaurateur (Institut des monuments culturels, Tirana); Ornela Durmishaj, animatrice du patrimoine (Parku arkeologjik kombëtar Bylis); Admir Xhelaj, restaurateur (Parku arkeologjik kombëtar Apollonia);

- du côté franco-canadien : Pascale Chevalier, UBP-Clermont-Ferrand 2, UMR 6298-ARTeHIS (chef de mission); Nicolas Beaudry, université du Québec à Rimouski (responsable de l'étude du quartier épiscopal); Tony Koželj et Manuela Wurch-Koželj, architectes (École française d'Athènes); Marie-Patricia Raynaud, UMR 8167-Orient et Méditerranée; Jean-Pierre Sodini, professeur émérite (université Paris 1); Coraline Vinos-Poyo, géomorphologue (université de Montpellier) ; Jean Cantuel, archéozoologue (université de Montpellier) ; Emmanuelle Fournier, docteur en archéologie (UBP) ; Julie Viriot, étudiante (UBP) ; Julien Bruyère et Sylvain Grosfilley, étudiants (université de Saint-Étienne); Jessica Laguë, étudiante (UQAR); Leonora Saliju, étudiante (université de Fribourg, Suisse) ; et Nadia Saint-Luc.

Des analyses ont été réalisées en France et au Canada, par Stéphane Büttner, géoarchéologue (Centre d'études médiévales Saint-Germain, Auxerre, UMR 6298); Manon Savard et C. Gagné, paléo-environnementalistes (UQAR).

3. N. CEKA et S. MUÇAJ, Byllis. Histoire et monuments, Tirana, 2011, p. 44-45.

4. Corpus Inscriptionum Latinarum, t. 3/1 (Inscriptiones Asiae, provinciarum Europae Graecarum, Illyrici Latinae), éd. Th. MOMMSEN, Berlin, 1873, $\mathrm{n}^{\circ} 600$; voir en dernier lieu R. HAENSCH et P. WEISS, « Ein schwieriger Weg. Die Straßenbauinschrift des M. Valerius Lollianus aus Byllis», Roemische Mitteilungen, 118, 2012 (sous presse).

5. Cette citerne rupestre à bouche rectangulaire - la seule du site (toutes les autres étant piriformes et à bouche ronde) - résulterait du surcreusement d'un bassin de foulage du raisin, désaffecté peut-être lors de l'aménagement du chai $\mathrm{N}$ en contrebas au nord-ouest. La petite production vinicole jusque-là dirigée vers le cardo, au pied du rempart de Viktôrinos, aurait été ainsi rapprochée de la cathédrale.

6. N. BEAUDRY, « Një punishte për prodhimin e verës në Bylisi », Monumentet, 28 (2010), Tirana, p. 41-50. 
7. Sur la présence d'escaliers dans les cuves de vinification, cf. J.-P. BRUN, «La discrimination entre les installations oléicoles et vinicoles ", in M-C. AMOURETTI et J-P. BRUN (éd.), La production du vin et de l'huile en Méditerranée, Bulletin de correspondance hellénique [BCH], suppl. 26, Athènes, 1993, p. 529.

8. Depuis, il n'a fait l'objet que de nettoyages et de quelques sondages rapides en 2002, peu profonds et destinés exclusivement à vérifier des détails architecturaux ponctuels. Ces sondages ont été menés sous la direction de S. Muçaj, cf. BCH, 127/2 (2003), p. 630-631.

9. La pièce est traversée par l'arasement de la façade sud du decumanus hellénistique, comprenant un seuil.

10. Le tracé du canal en usage à l'époque paléochrétienne tardive n'est plus parallèle aux alignements des murs d'épaisseur hellénistique, mais toujours quasiment au centre du decumanus hellénistico-romain. La construction visible remplace probablement le cloaque antique.

11. Byllis 3 rassemblera les études de matériels archéologiques (céramique, verre, métal, etc.).

12. N. CEKA et S. MUÇAJ, Byllis. Histoire et Monuments, Tirana, 2011.

13. Qui a soutenu en mai 2011 à l'université de Tirana (Faculté d'histoire et de philologie) une thèse de doctorat d'archéologie de l'Antiquité tardive intitulée «Instalimet litugjike në kishet paleokristiane dhe vazhdimësia e tyre në mesjetë ne Shqipëri » (Installations liturgiques des églises paléochrétiennes et médiévales en Albanie).

14. La base de données PostGre SQL est accessible via le portail http://adriaticummare.org/.

INDEX

Mots-clés : quartier épiscopal

Index géographique : Albanie/Byllis 